\title{
Torasemide reduces dilated cardiomyopathy, complication of arrhythmia, and progression to heart failure
}

\author{
L.N. Han ${ }^{1}$, S.L. Guo ${ }^{2}$, X.M. Lin $^{3}$, X.M. Shi ${ }^{1}$, C.B. Zang ${ }^{1}$, L.M. Yang ${ }^{1}$ and \\ G.L. Ding ${ }^{1}$
}

${ }^{1}$ First Department of Cardiovascular Internal Medicine in South Building, PLA General Hospital, Beijing, China

${ }^{2}$ School of Automation, Beijing Institute of Technology, Beijing, China

${ }^{3}$ Department of Cardiovascular Internal Medicine, PLA 187 Center Hospitals, Haikou Province, China

Corresponding authors: S.L. Guo / X.M. Lin

E-mail: guoshuli@bit.edu.cn / lndoccn@163.com

Genet. Mol. Res. 13 (3): $7262-7274$ (2014)

Received July 10, 2013

Accepted August 6, 2014

Published September 5, 2014

DOI http://dx.doi.org/10.4238/2014.September.5.11

\begin{abstract}
The aim of this study was to analyze the incidence and types of arrhythmia and their relationship with the severity and prognosis of chronic heart failure $(\mathrm{CHF})$ in patients with dilated cardiomyopathy (DCM), and to investigate the therapeutic effect of torasemide versus furosemide on $\mathrm{CHF}$ and incidence of arrhythmia. DCM patients with NYHA cardiac function II-IV were continuously monitored using a 24-h dynamic electrocardiogram (Holter), and arrhythmia incidence was analyzed by computer automatic analysis combined with manual assessment. In total, 125 participants were evenly divided into two groups: torasemide group which received 10 mg oral torasemide once daily) and regular anti-heart failure treatment $(\mathrm{N}=65)$, and furosemide group which received torasemide (20 mg once daily orally) and regular antiheart failure treatment $(\mathrm{N}=60)$. Another 60 normal healthy persons served as the normal control group.
\end{abstract}


Incidence and severity of arrhythmia increased when degree of CHF was elevated. Size of left atrium was related to atrial fibrillation and size of left ventricle was related to malignant arrhythmia. At 3 months after treatment, cardiac function in both groups improved and incidence and severity of arrhythmia in both groups were reduced. However, left ventricular ejection fraction (LVEF) was higher in the torasemide group than in the furosemide group, while incidence of arrhythmia was lower in the torasemide group. Arrhythmias frequently occurred in patients with DCM and HF. Type of cardiac arrhythmia is closely related to ventricular enlargement and cardiac function grade. Torasemide is better for improving cardiac function to reduce arrhythmia and CHF compared to furosemide.

Key words: Dilated cardiomyopathy; Heart failure; Arrhythmia; Torasemide; Furosemide

\section{INTRODUCTION}

DCM (dilated cardiomyopathy) is a progressive disease characterized by an increase in diameter and volume of the left or both ventricles, leading to progressive dilatation and impaired systolic function that is not secondary to or cannot be exclusively justified by abnormal loading conditions (e.g., valve disease, hypertension) or by concomitant coronary artery disease, and it represents the most common form of non-ischemic cardiomyopathy (Hay et al., 2004). Five-year survival rate for patients with DCM is less than $50 \%$ and the mortality rate is higher. Complicated or severe arrhythmias are associated with total cardiac mortality rate and may turn out to be the cause of death. The TORIC (Torasemide In Congestive Heart Failure) study, an open-label study of patients with New York Heart Association class II to III HF comparing furosemide with torasemide showed that a greater proportion of patients receiving torasemide improved their functional class and that fewer patients receiving torasemide died (Cosin and Diez, 2002). Additionally, a meta-analysis of the existing studies has suggested trends toward improved functional status and mortality with torasemide compared with furosemide (DiNicolantonio, 2012). Torasemide, a newer type of loop diuretic, is important for the symptomatic treatment of CHF. We hypothesize that torasemide improves heart functional class in a greater proportion of patients compared with furosemide, which may be associated with significantly better alleviation off arrhythmia. Therefore, we designed this study to investigate the relationship between severity of arrhythmia and heart function and effect of torasemide treatment on arrhythmia, cardiac function and prognosis compared with furosemide.

\section{MATERIAL AND METHODS}

\section{General information}

In this study, 125 patients with DCM seen at PLA General Hospital were retrospectively analyzed. Patients were diagnosed with DCM according to the 1995 WHO/ISFC 
diagnostic criteria for DCM (Richardson et al., 1996), of whom 69 cases were male and 56 cases female. Patients' age ranged from 25 to 73 years old, with a mean of 58.9 years old. Three patients were 30 years old and younger, 18 cases were 31 to 50, 82 cases were 51 to 70 , and 21 cases were older than 70. Cardiac functional grading standards were according to the New York Heart Association (NYHA) cardiac function classification (Ganiats et al., 1998). Another 60 healthy persons served as the normal control group. These three groups were recruited in matched pairs with the same age, gender and NYHA class. The patients were evaluated by cardiovascular X-ray examination, 12-lead electrocardiograms (ECG), 24-h dynamic ECG with Beijing Century Jinke Medical Instrument Limited Company Dynamic Electrocardiogram Series MIC-3H-3 (Beijing, China), echocardiography examination, and coronary angiography. Peripheral blood electrolytes were determined when necessary to exclude acute viral myocarditis, rheumatic heart disease, coronary heart disease, congenital cardiovascular disease and various secondary cardiomyopathies. The patients were administered anti-heart failure treatment including angiotensin-converting enzyme inhibitor (ACEI) fosinopril $10 \mathrm{mg}$ once daily orally, beta-blocker metoprolol tartrate tablets $12.5 \mathrm{mg}$ twice daily orally, potassium-sparing diuretic spironolactone $20 \mathrm{mg}$ once daily orally, and cardiotonic digoxin $0.125 \mathrm{mg}$ once daily orally. The patients were divided into two balanced groups, torasemide group $(\mathrm{N}=65)$ who received torasemide 20 $\mathrm{mg}$ twice daily and the regular anti-heart failure treatment mentioned above for 12 months and furosemide group $(\mathrm{N}=60)$ who received furosemide $20 \mathrm{mg}$ twice daily and the regular anti-heart failure treatment for 12 months. When system of HF was relieved, administration of torasemide and furosemide was changed to once every two days or twice a week. The participants were followed up with 24-h dynamic electrocardiogram, color Doppler echocardiography examination, measurement of $\mathrm{N}$-terminal probrain natriuretic peptide (NT-proBNP) and C-reactive protein (CRP) and 6-minute walking distance test (6MWT) before treatment and 12 months after treatment. Besides safety and tolerability, efficacy was assessed by documentation of mortality, morbidity, functional class and serum potassium levels every 3 months. This study was conducted in accordance with the Declaration of Helsinki, and with approval from the Ethics Committee of the Chinese PLA General Hospital. Written informed consent was obtained from all participants.

\section{4-h dynamic ECG}

Every patient was monitored with the Dynamic Electrocardiogram Series MIC-3H-3 for 23-24 h. Recorded data were input in a computer and human-computer dialogue was made to draw 24-h arrhythmia conclusion.

Ventricular arrhythmias were classified by the Lown standard. Diagnosis criteria of Lown III was complex ventricular arrhythmia, continuous ventricular premature contraction 3 times. Duration of ventricular arrhythmia less than $30 \mathrm{~s}$ was non-sustained ventricular tachycardia. Duration of more than $30 \mathrm{~s}$ was sustained ventricular tachycardia.

\section{Color Doppler echocardiography examination}

Patients' left ventricular end diastolic diameter (LVEDd) was measured using Philips IU22 echocardiography (Philips, Amsterdam, Netherlands) with a transducer frequency of 2.5 
$\mathrm{MHz}$ at parasternal short axis view. LVEDd was measured at end diastolic period for 3 consecutive heartbeat cycles and its average value was calculated and considered as the LVEDd value. Left atrium diameter (LAD) was measured in left ventricular long axis at left ventricular end systolic period.

Diagnostic criteria of left ventricular enlargement for males was LVEDd more than $55 \mathrm{~mm}$ and for females more than $50 \mathrm{~mm}$. Diagnostic criterion of left atrium enlargement for males was LAD more than $33 \mathrm{~mm}$ and for females LAD more than $30 \mathrm{~mm}$.

\section{Determination of plasma CRP and serum NT-proBNP}

Blood samples were obtained from all participants in the recumbent position from an antecubital vein. A total of $4 \mathrm{~mL}$ blood was drawn into two vacuum tubes containing 0.3 $\mathrm{mL}$ sodium heparin and coagulant, respectively. Serum levels of CRP were determined by immunoturbidimetry using a Siemens BN II autoanalyzer (Siemens, Erlangen, Germany) in the Department of Biochemistry in Hainan Branch of Chinese PLA General Hospital. A detection kit was also purchased from Siemens. The plasma level of NT-proBNP was detected by a heterogeneous immunoassay using a Siemens RXL-MAX autoanalyzer (Siemens). The procedure was according to manufacturer specifications.

\section{6-minute walking distance test}

All patients were instructed to walk as far as possible for $6 \mathrm{~min}$ in a straight corridor and their heart and rhythm were monitored with a distance-controlled ECG monitor. The distance covered in that time period was recorded.

\section{Statistical analysis}

The SPSS 11.0 statistical software was used for statistical analysis. Measurement data are reported as means $\pm \mathrm{SD}$. A chi-square test was used for comparison of categorical data such as number of arrhythmias and incidence rate of malignant ventricular arrhythmia. Comparisons of LAD and LVEF between two groups were done with $t$-test. Comparisons of mean LVEDD, LVEF, 6MWT, NT-proBNP and CRP between three groups were carried out using simple factor analysis of variance and comparisons between two groups were with a $t$-test. Mortality between treatment groups adjusting for NYHA class at baseline was analyzed by logistic regression. $\mathrm{P}<0.05$ was considered to be statistically significant.

\section{RESULTS}

\section{Participants' cardiac function characteristics before treatment}

Participants were enrolled in a 12-month, open-label, parallel study and were randomized either to continue on their stable $10 \mathrm{mg} /$ day dose of oral torasemide or $20 \mathrm{mg}$ /day dose of furosemide. Echocardiography showed that there was right atrial enlargement in 7 cases (5.6\%), right ventricular enlargement in 36 cases $(28.8 \%)$, left atrial enlargement in 42 cases (33.6\%), and left ventricular enlargement in 98 cases (78.4\%). Ventricular wall movements 
were commonly weakened in all cases. Color echocardiography showed that LVEDd ranged from 56 to $78 \mathrm{~mm}$, with a mean of $62 \mathrm{~mm}$, and LAD ranged from 36 to $55 \mathrm{~mm}$, with a mean of $45 \mathrm{~mm}$. Based on the New York Heart Association (NYHA) classification of cardiac function, there were 41 cases of cardiac function II (32.8\%), and 44 and 40 cases of III and IV, respectively $(67.2 \%)$.

\section{Incidence rate and classification of cardiac arrhythmia before treatment}

Incidence of arrhythmia in the cardiac function II group was $82.9 \%$, and in III and IV groups, it was $97.6 \%$, respectively. Arrhythmia occurred in 111 cases $(92.9 \%)$. The incidence of atrial arrhythmia was the highest, next was ventricular arrhythmias, and then transduction block. Incidence of atrial arrhythmia (atrial premature beats, paroxysmal atrial tachycardia and atrial fibrillation) was 64 cases (51.2\%), ventricular arrhythmia (premature ventricular contractions, paired ventricular extrasystoles, non-sustained ventricular tachycardia) was 58 cases (46.4\%), and transduction block (sinoatrial block, atrioventricular block, intraventricular block) was 13 cases (10.4\%).

\section{Relationship between occurrence rate of arrhythmia type and cardiac function}

Incidence rate of one type of arrhythmia in heart function II and III-IV was 7.3 and $1.1 \%$, respectively. Incidence rate of two types of arrhythmia in heart function II and III-IV was 19.5 and $14.2 \%$. Incidence of three types of arrhythmias in heart function II and III-IV was 51.2 and $26.2 \%$. Incidence of more than four types of arrhythmias in heart function II and III-IV was 12.2 and $47.6 \%(\mathrm{P}<0.01)$. The findings above indicated that the worse left ventricular function was, the more complex arrhythmias were. Occurrence rate of arrhythmia was not statistically significant in different functional levels, but arrhythmia was closely related to heart failure level. The higher the level of HF was, the more types of arrhythmia occurred. Incidence rate of arrhythmia in different heart function levels are shown in Table 1.

Table 1. Incidence of arrhythmia in different cardiac function level.
$\begin{aligned} & \text { NYHA heart } \\
& \text { function }\end{aligned}$
\begin{tabular}{lcccccc} 
Case (N) & $\begin{array}{c}\text { Incidence of } \\
\text { arrhythmia (N, \%) }\end{array}$ & One type (N, \%) & Two types (N, \%) & Three types (N, \%) & $\begin{array}{c}\text { Four types and } \\
\text { more than (N, \%) }\end{array}$ \\
\hline II & 41 & $35(82.9)$ & $3(7.3)$ & $8(19.5)$ & $21(51.2)$ & $5(12.2)$ \\
III, IV & 84 & $82(97.6)$ & $1(1.1)$ & $12(14.2)$ & $22(26.2)^{* *}$ & $40(47.6)^{* *}$ \\
\hline
\end{tabular}

NYHA $=$ New York Heart Association. *Indicated $\mathrm{P}<0.05$ vs heart function $\alpha$ group, $* *$ indicated $\mathrm{P}<0.01 v s$ heart function.

\section{Relationship between size of left atrium and atrial fibrillation}

Among 125 patients with DCM, 39 cases had atrial fibrillation and 86 patients had no atrial fibrillation. The level of LAD and LVEF between the group with atrial fibrillation and the group without atrial fibrillation had significant difference. In detail, atrial fibrillation incidence significantly increased with enlarged LAD $(\mathrm{P}<0.01)$ and decreased LVEF $(\mathrm{P}<$ 0.01). Relationships between atrial fibrillation and age, LAD and LVEF are shown in Table 2 and Figure 1. 


\section{Relationship between size of left ventricle and incidence of malignant ventricular arrhythmia}

A total of 58 cases suffered ventricular arrhythmia, among whom LVEDd of 34 cases was more than $50 \mathrm{~mm}$. In the three groups, the occurrence rates of malignant ventricular arrhythmias were 5 cases (11.6\%), 11 cases (31.4\%), 18 cases $(47.4 \%)$. Incidence of malignant ventricular arrhythmia in two later groups was extremely significantly increased compared with the first group (LVEDd 50-59mm) $(\mathrm{P}<0.01)$, while the following two groups (LVEDd $60-69 \mathrm{~mm}$ and $\geq 70 \mathrm{~mm}$ ) was without significant difference. Incidence of malignant ventricular arrhythmia in different groups of LVEDd are shown in Table 3.

\section{Participants' heart function in two groups before therapy}

Table 2. Incidence of arrhythmia in different cardiac function level.

\begin{tabular}{lclll}
\hline Group & No. of case $(\mathrm{N})$ & Age & LAD $(\mathrm{mm})$ & LVEF (\%) \\
\hline Atrial fibrillation & 39 & $58 \pm 12$ & $45 \pm 4$ & $30 \pm 11$ \\
No atrial fibrillation & 86 & $53 \pm 10$ & $31 \pm 3^{* *}$ & $42 \pm 12^{* *}$ \\
\hline
\end{tabular}

$\mathrm{LAD}=$ left atrium diameter; $\mathrm{LVEF}=$ left ventricular ejection fraction. $* * \mathrm{P}<0.01$ vs atrial fibrillation group. LAD indicated left atrial diameter. LVEF indicated left ventricular ejection fraction.
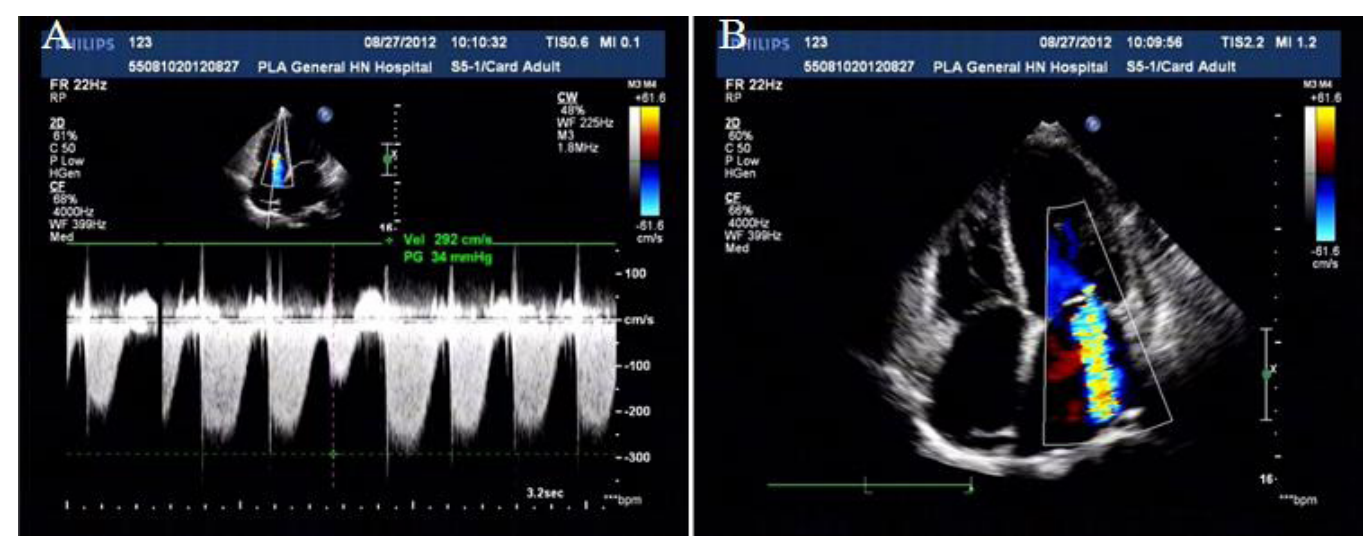

Figure 1. (A) Four-chamber view of transthoracic color Doppler echocardiography showed that tricuspid valve moderates regurgitation. Spectrum image indicated pulmonary artery high pressure and atrial fibrillation. (B) Fourchamber view of transthoracic color Doppler echocardiography showed that bicuspid valve moderate regurgitation and pericardial effusion.

Table 3. Relationship between LVEDd and incidence rate of malignant ventricular arrhythmia.

\begin{tabular}{lcccc}
\hline LVEDd $(\mathrm{mm})$ & $\mathrm{N}$ & Lawn's classification $\geq 3$ level $(\mathrm{N}, \%)$ & Lawn's classification $<3$ level $(\mathrm{N}, \%)$ & $\mathrm{P}$ \\
\hline$<50$ & $9(7.2 \%)$ & 0 & $9(6.9)$ & $<0.01$ \\
$50-59$ & $43(34.4 \%)$ & $5(11.6)$ & $38(88.4)$ & \\
$60-69$ & $35(28.0 \%)$ & $11(31.4)$ & $24(68.6)$ & \\
$\geq 70$ & $38(30.4 \%)$ & $18(47.4)$ & $20(52.6)$ & \\
\hline
\end{tabular}

LVEDd $=$ left ventricular end diastolic diameter. 
There were no differences in age, New York Heart Association function class, LVEDD, LVEF, 6MWT, NT-proBNP and CRP in the pre-existing CHF therapy between the study groups $(\mathrm{P}>0.05)$ (Table 4). Of the 125 patients included in this analysis, 65 patients received torasemide $10 \mathrm{mg} /$ day orally, with 60 of these patients receiving spironolactone. Sixty patients received furosemide at a dose of $20 \mathrm{mg}$ /day orally, of which 45 patients were taking other diuretics, 24 the potassium-sparing diuretic spironolactone, and 21 thiazides.

\begin{tabular}{|c|c|c|c|}
\hline & Torasemide group $(\mathrm{N}=65)$ & Furosemide group $(\mathrm{N}=60)$ & Normal control group $(\mathrm{N}=60)$ \\
\hline Age (means \pm SD) & $58.2 \pm 9.5$ & $59.4 \pm 10.2$ & $59.3 \pm 9.8$ \\
\hline Gender male/female & $38 / 27$ & $34 / 26$ & $33 / 27$ \\
\hline Weight (kg) & $81 \pm 7$ (male) & & \\
\hline $59 \pm 5$ (female) & $80 \pm 6$ (male) & & \\
\hline $59 \pm 6$ (female) & $82 \pm 7$ (male) & & \\
\hline \multicolumn{4}{|l|}{$60 \pm 5$ (female) } \\
\hline Height $(\mathrm{cm})$ & $168 \pm 4$ (male) & & \\
\hline $158 \pm 3$ (female) & $169 \pm 4$ (male) & & \\
\hline $157 \pm 4$ (female) & $167 \pm 4$ (male) & & \\
\hline \multicolumn{4}{|l|}{$157 \pm 3$ (female) } \\
\hline Heart failure (NYHA) (II/III/IV) & $22 / 21 / 22$ & $19 / 20 / 21$ & $0 / 0 / 0$ \\
\hline \multicolumn{4}{|l|}{ Concomitant heart disease } \\
\hline Hypertension & 8 & 6 & \\
\hline Coronary heart disease & 7 & 6 & \\
\hline Valvupathy & 3 & 3 & \\
\hline \multicolumn{4}{|l|}{ Medication } \\
\hline ACEI & $65(100 \%)$ & $60(100 \%)$ & \\
\hline Beta blockade & $51(78.4 \%)$ & $47(78.3 \%)$ & \\
\hline Digitalis & $14(21.5 \%)$ & $13(21.7 \%)$ & \\
\hline Spitonolactone & $60(92.3 \%)$ & $24(40.0 \%)$ & \\
\hline Incidence of arrhythmia & $3.5 \pm 0.5^{* *}$ & $3.7 \pm 0.6^{* *}$ & $1.3 \pm 0.5$ \\
\hline LVEDd (mm) & $62 \pm 5^{* *}$ & $61 \pm 4^{* *}$ & $43 \pm 4$ \\
\hline LVEF (\%) & $39.4 \pm 3.5^{* *}$ & $40.2 \pm 3.2 * *$ & $56.6 \pm 3.4$ \\
\hline 6MWT (m) & $286 \pm 12^{* * *}$ & $278 \pm 11^{* *}$ & $425 \pm 17$ \\
\hline NT-proBNP (pg/mL) & $864 \pm 97 * *$ & $873 \pm 110^{* *}$ & $52 \pm 12$ \\
\hline $\mathrm{CRP}(\mathrm{mg} / \mathrm{dL})$ & $5.63 \pm 0.56 * *$ & $5.76 \pm 0.59 * *$ & $0.27 \pm 0.08$ \\
\hline
\end{tabular}

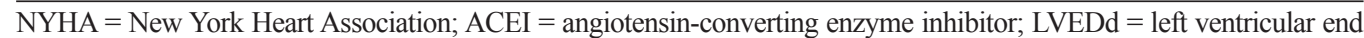
diastolic diameter; $\mathrm{LVEF}=$ left ventricular ejection fraction; $6 \mathrm{MWT}=6$-minute walking distance test; NT-proBNP = $\mathrm{N}$-terminal probain natriuretic peptide; $\mathrm{CRP}=\mathrm{C}$-reactive protein. Data are reported as means $\pm \mathrm{SD} . * * \mathrm{P}<0.01$.

\section{Follow-up and prognosis}

All participants were followed up every 3 months except for emergency. If the symptom of HF was alleviated, the oral dose of diuretic was decreased or stopped. The average terminal follow-up duration was 12.3 months. At an average of 20 days of hospitalization, lower levels of NT-proBNP, CRP and body weight and higher level of drop of input and output indicated therapeutic effect in both treatments. But there was no difference between these two groups (Table 5). At 12.3 months follow-up, the two treatment groups were associated with lower levels of NT-proBNP, CRP, LVEDD, readmission and morality, but higher LVEF and 6-MWT. However, levels of NT-proBNP CRP, LVEDD, readmission and mortality were lower, and levels of LVEF and 6-MWT were higher compared with the furosemide group (Figure 2). Indication of rehospitalization was acute heart failure attack, shortness of breath, limb edema, and paroxysmal nocturnal dyspnea, with or without coughing up pink frothy sputum. 
Table 5. Cardiac function in two groups pre-treatment and post-treatment.

\begin{tabular}{|c|c|c|c|c|c|c|c|c|}
\hline \multirow[t]{2}{*}{ Torasemide group } & \multicolumn{8}{|c|}{ Furosemide group } \\
\hline & Pre-treatment & 20 days Post-treatment & 12 months Post-treatment & $P$ & Pre-treatment & 20 days Post-treatment & 12 moths Post-treatment & $P$ \\
\hline NT-proBNP (pg/mL) & $864 \pm 97$ & $367 \pm 76$ & $146 \pm 35$ & $<0.01$ & $873 \pm 110$ & $432 \pm 72^{\# \#}$ & $201 \pm 57^{\# \#}$ & $<0.01$ \\
\hline $\mathrm{CRP}(\mathrm{mg} / \mathrm{dL})$ & $5.7 \pm 0.6$ & $2.7 \pm 0.4$ & $1.5 \pm 0.3$ & $<0.01$ & $5.6 \pm 0.5$ & $3.4 \pm 0.4^{\# \#}$ & $2.8 \pm 0.3^{\# \#}$ & $<0.01$ \\
\hline Potassium (mM) & $4.1 \pm 0.6$ & $4.0 \pm 0.5$ & $3.9 \pm 0.5$ & $>0.05$ & $4.0 \pm 0.5$ & $4.0 \pm 0.4^{\star}$ & $3.9 \pm 0.4^{\star}$ & $>0.05$ \\
\hline LVEDd (mm) & $63 \pm 8$ & $56 \pm 7$ & $52 \pm 7$ & $<0.01$ & $62 \pm 9$ & $57 \pm 7 \Perp$ & $55 \pm 8^{\#}$ & $<0.01$ \\
\hline LVEF (\%) & $33.4 \pm 3.5$ & $43.5 \pm 4.4$ & $50.6 \pm 5.4$ & $<0.01$ & $32.2 \pm 3.3$ & $41.3 \pm 4.2^{\#}$ & $46.3 \pm 4.6^{\# \#}$ & $<0.01$ \\
\hline 6MWT (m) & $286 \pm 12$ & $336 \pm 23$ & $384 \pm 46$ & $<0.01$ & $278 \pm 11$ & $331 \pm 21^{\wedge}$ & $367 \pm 32^{\# \#}$ & $<0.01$ \\
\hline Sodium (mM) & $142 \pm 3$ & $140 \pm 4$ & $142 \pm 3$ & $>0.05$ & $143 \pm 4$ & $141 \pm 3^{4}$ & $140 \pm 5^{\wedge}$ & $>0.05$ \\
\hline $\begin{array}{l}\text { Drop of input and } \\
\text { output }(\mathrm{mL})\end{array}$ & & $2123 \pm 122$ & & & & $2011 \pm 138^{\#}$ & & \\
\hline \multirow[t]{2}{*}{ Body weight $(\mathrm{kg})$} & $81 \pm 7(\mathrm{~m})$ & $78 \pm 6(\mathrm{~m})^{*}$ & & & $80 \pm 6(\mathrm{~m})$ & $77 \pm 5(\mathrm{~m})^{\star} * *$ & & \\
\hline & $59 \pm 5(f)$ & $57 \pm 4(\mathrm{f})^{*}$ & & & $59 \pm 6(f)$ & $57 \pm 4(\mathrm{f})^{\mathbf{4} *}$ & & \\
\hline Arrhythmia (N) & $4.5 \pm 1.2$ & & $2.4 \pm 0.9^{* *}$ & & $4.6 \pm 1.3$ & & $2.9 \pm 1.0^{* * * \#}$ & \\
\hline Readmission (N) & $4(6.5 \%)$ & & & & $6(10 \%)^{\# \#}$ & & & \\
\hline Morality (\%) (N) & $1(1.5 \%)$ & & & & $2(3.3 \%)^{\#}$ & & & \\
\hline
\end{tabular}

Data are reported as means $\pm \mathrm{SD} . * \mathrm{P}<0.05, * * \mathrm{P}<0.01$ vs pre-treatment. ${ }^{\#} \mathrm{P}<0.5 ;{ }^{*} \mathrm{P}<0.01 ;{ }^{\wedge} \mathrm{P}>0.05$ vs Torasemide group. For abbreviations, see legend to Table 4.
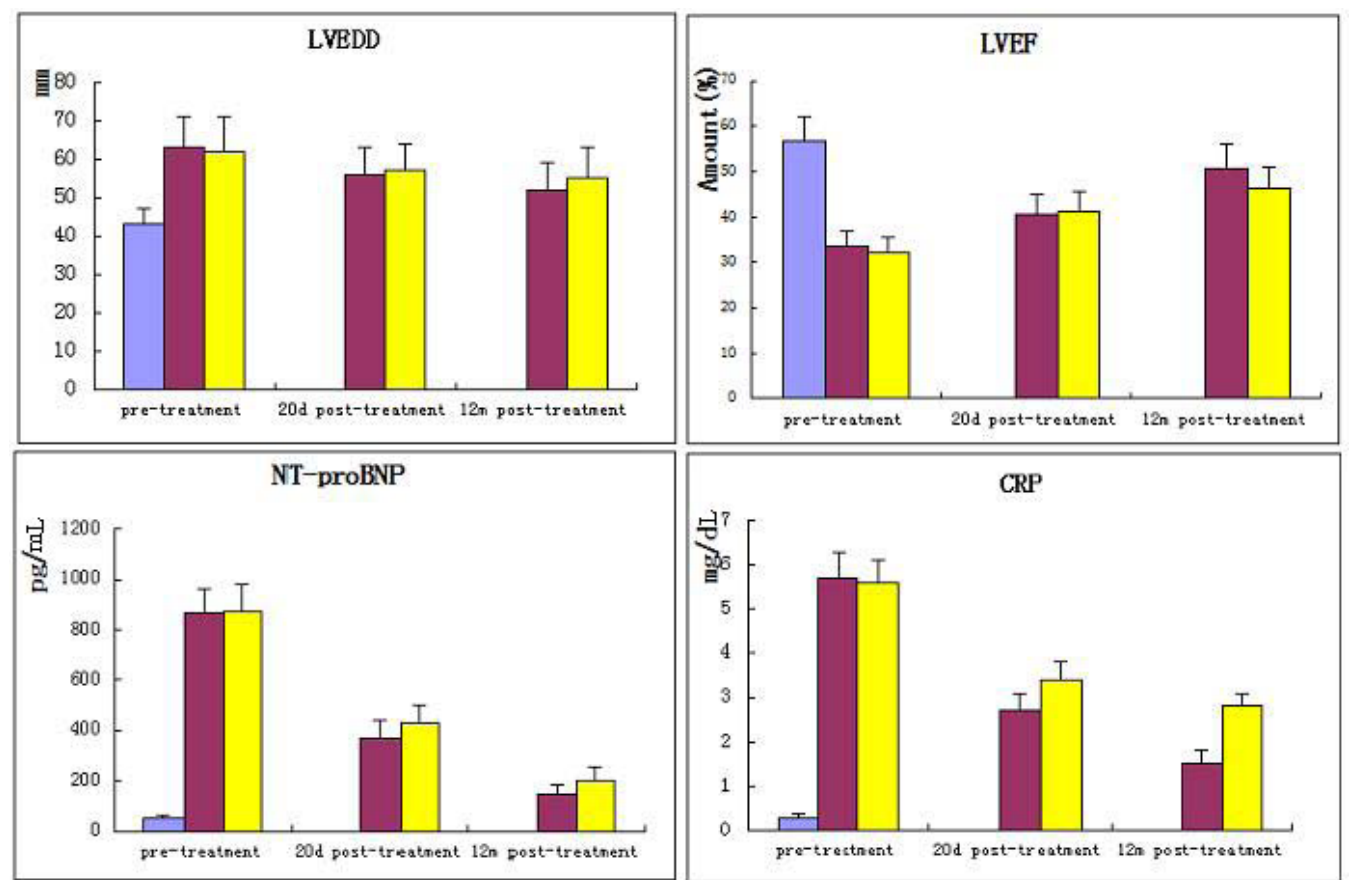

Figure 2. Cardiac function in both groups before and after treatment. LVEDd $=$ left ventricular end diastolic diameter; $\mathrm{LVEF}=$ left ventricular ejection fraction; $\mathrm{NT}$-proBNP $=\mathrm{N}$ terminal pro brain natriuretic peptide; $\mathrm{CRP}=$ $\mathrm{C}$-reactive protein. There was no difference in cardiac function in the pre-existing CHF therapy between the study groups. At average 20 days in hospitalization, lower levels of NT-proBNP, CRP, LVEDd, higher level of LVEF were found in both treatment groups. At 12.3 months follow-up, two treatment groups were also associated with lower level of NT-proBNP, CRP, LVEDd, but higher LVEF. But levels of NT-proBNP, CRP, LVEDd, were lower. Level of LVEF was higher compared with furosemide group. 


\section{DISCUSSION}

DCM is currently classified as primary (genetic, acquired, or mixed), secondary (e.g., infiltrative or autoimmune), and idiopathic. Genetic inheritance is likely to play a role in the development of the disease in 20 to $35 \%$ of patients, but also acquired conditions such as metabolic abnormalities, inflammatory and infectious processes, neuromuscular diseases and a large variety of cardiotoxic agents (chemotherapeutic agents, alcohol, illicit drugs) can lead to DCM (Slavich et al., 2011). DCM onset is slow and can appear at any age, with most patients being 30 to 50 years old. In some patients, DCM is accompanied by primary hypertension. Yearly diagnostic rate is approximately 8 of 10 million, while the prevalence rate is about 37 of 10 million, in which 33.3\% of patients have cardiac function III or IV (Okutucu and Oto, 2010). About $20 \%$ of DCM patients have a family history of cardiomyopathy (Yoskovitz et al., 2012).

It has recently become clear that gene mutations in various structural proteins such as those encoding cytoskeletal, nucleoskeletal, mitochondrial, and calcium-handling proteins can contribute to the development of DCM (Serio et al., 2012). The common unifying theme of these regions is that genetic defects leading to ineffective contraction cause ventricular remodeling, ultimately leading to cardiomyopathy. For example, genes encoding the thick filament components myosin heavy chain 7 (MYH7) and myosin-binding protein C (MYBPC3) were reported to be linked to inherited DCM (McNally et al., 2013). The displacement of endogenous mammalian Enabled, actin cytoskeletal signal modulators, and vasodilator-stimulated phosphoprotein proteins, actin cytoskeletal signal modulators lead to weakness of cardiac in force production (Eigenthaler et al., 2003). Besides abnormal expression of gene, change in protein also takes part in the process of DCM such as Fas protein (Wei et al., 2012).

Some indicators of HF, such as atrial natriuretic peptide and brain natriuretic peptide, have been found to be more closely correlated with cardiac enlargement than CHF or lung edema (Sugihara et al., 2013; Lourenco et al., 2010). CRP levels are elevated in CHF and increasing levels are associated with increasing morbidity and mortality in ischemic and non-ischemic etiologies (Nakagomi et al., 2010). In this study, we found higher levels of NT-proBNP and CRP in the two DCM groups than in the normal control group, and in our previous study, NT-proBNP and CRP levels were negatively correlated with LVEF (Han et al., 2013). The DCM heart presumably undergoes a complex series of changes in neurohumoral mechanisms such as the sympathetic nervous and renin-angiotensin-aldosterone systems to compensate for the reduction in cardiac contractility, which are likely to affect cardiac gene expression resulting in electrical remodeling, ultimately evolving to cardiac arrhythmia, pump failure, and sudden death (Tsutamoto et al., 2011). Additionally, excessive angiotensin from the activation of the sympathetic nervous system and renin-angiotensin-aldosterone systems is involved in the excretion of potassium and magnesium into the urine, resulting in unstable cardiac electrical activity, easily producing proarrhythmia.

Clinical traits, both cardiac and extracardiac, may recur in association with DCM phenotype. The former include conduction defects, structural abnormalities such as left ventricular noncompaction and recurrence of atrial or ventricular arrhythmias; the latter commonly affect the musculoskeletal, ocular, auditory, nervous, and integument systems. Thus, the complexity of DCM arrhythmia is greater compared to any other cardiomyopathy (Gosselin-Badaroudine et al., 2012). The myocardium may be arrhythmogenic before or during development of HF, though at present, it remains unclear how HF and arrhythmogenic 
changes proceed in carriers of DCM mutations. Biopsy has confirmed that about $70 \%$ of DCM patients have multiple subendocardial diffuse, fibrous, patchy and gross scars in the left ventricle, which are regarded as potentially arrhythmogenic (Dickerson et al., 2013). The main micro features of DCM are myocyte elongation, myocardial apoptosis, and hypertrophy of the relic myocytes. Additionally, there is an excessive collagen deposition and decreased capillary density, with both reactive (interstitial and perivascular) and reparative patterns of fibrosis, which is considered the result of damage due to microvascular ischemia and myocardial wall inflammation. The cellular and extracellular changes result in uneven depth of the myocardial wall. Difference in excitability and conductivity between the myocardial injury area, fibrotic cardiac scar and normal cardiac area supply a pathologic basis for unidirectional block and conduction delay, finally leading to reentrant arrhythmias. Recent demonstration and continuous expansion of knowledge regarding arrhythmogenesis genes in DCM open a new field of diagnosis and prognosis of these patients. For example, gadolinium enhancement (Koutalas et al., 2013), LMNA (lamin A/C) gene mutations (Sun et al., 2010), sodium channels SCN5A Arg222Gln missense variant (Mann et al., 2012), and cardiac ryanodine receptor $\mathrm{NH}_{2}$-terminal mutation (Tang et al., 2012) are closely related to ventricular arrhythmogenesis. Moreover, matrix metalloproteinase 9, tissue inhibitor of matrix metalloproteinase, procollagen type I carboxyterminal peptide, procollagen type III aminoterminal peptide, and their ratio have been found to be indicative of a deranged equilibrium in myocardial collagen deposition/degradation and collagen I/III synthesis which are related to ventricular arrhythmogenesis (Flevari et al., 2012). Elucidation of the genetic basis of familial DCM can enable effective gene-targeted therapy to be implemented. Also, multiple types of progressive electrical remodeling, such as ion channel reduction in Ito and Kv4.2 transcription and concurrent upregulation of $\mathrm{Na}^{+}-\mathrm{Ca}^{2+}$ exchanger-1, occur and the action potential duration is prolonged (Suzuki et al., 2012), thus the reason for arrhythmogenesis being complex.

This study showed that atrial arrhythmia was the most common species in DCM arrhythmia (51.2\%), followed by ventricular arrhythmias and conduction block. Maybe significantly expanded LAD increases atrial capacity load and internal pressure, so that arterial lumen intima was thickened and arteriolar was gradually occluded. Cardiac malnutrition results in atrial myocardial tissue becoming ischemic, degenerated, necrotic, and fibrotic. Electrical activity of injured atrium is inconsistent, so local conduction delays and effective refractory period increases. It is easy to form reentry, twists, turns and complex annular movement, ultimately leading to atrial fibrillation (Aleksova et al., 2010). Spontaneous activities were also confirmed as spontaneous contractions in papillary muscles from the left ventricle. Similar measurements were determined in the right ventricle and left atrial muscles. Myocardial automaticity appears to be considerably enhanced in the left ventricle (Suzuki et al., 2012).

This study showed that cardiac function classification level of HF was elevated, with arrhythmia incidence also increasing. Left ventricular function in patients with DCM is closely related to the occurrence of severe ventricular arrhythmias (Haugaa et al., 2012). Dilated left ventricle has a larger left atrial appendage and seems to be at a higher risk for thrombus formation (Bakalli et al., 2010). Patients with DCM and co-existing AF have shown a weaker effect of 3-hydroxy-3-methylglutaryl coenzyme A inhibitor concerning the reduction of IL-6 and NT-proBNP concentration to inhibit remodeling of the myocardium (Bielecka-Dabrowa et al., 2010). Conduction block occurred at a rate of up to $45 \%$ in DCM patients, including the atrioventricular node block and bundle branch block, because diffuse cardiac damage in DCM 
patients is involved in conduction system. Conduction block in patients with DCM is characterized as multiple types. Additionally, as the disease progresses, the ventricle is markedly enlarged and Purkinje fibers are elongated and damaged, which is the reason for cardiac impulse conduction obstacles (Pruszczyk et al., 2007; Koutalas et al., 2013). To prevent sudden death, an early genetic diagnosis and start of therapy may be important for individuals with a family history. Prophylactic implantable cardioverter defibrillator therapy combined with cardiac resynchronization has become standard treatment in DCM patients with complete left bundle branch block and mild to moderate heart failure despite optimal pharmacotherapy (Fujii and Takami, 2008; Grimm, 2012).

DCM is recognized as a significant cause of morbidity and mortality. DCM induced heart failure is one of the causes of death in patients with DCM. Atrial fibrillation and paroxysmal supraventricular tachycardia deteriorate hemodynamics and impair left ventricular function. Complications such as embolism also increase the cause of death in DCM patients. The risk factors for sudden cardiac death in DCM with implantable cardioverter defibrillator include asymptomatic nonsustained ventricular tachycardia and poor left ventricular function (Dimas et al., 2009). DCM patients complicated with atrial fibrillation have worse prognosis than the DCM patients with sinus rhythm (Aleksova et al., 2010).

Treatment strategies of DCM vary due to its diverse etiopathology ranging from myocardial infarction, myocarditis and pressure overload, etc. Diuretic therapy is a core element in the treatment of CHF because diuretics relieve cardiac load by reducing water retention. Torasemide or 1-isopropyl-3-\{[4-(3-methyl-phenylamino) pyridine]-3- sulfonyl $\}$ urea, a new longacting loop diuretic, combined with spironolactone, is widely used in DCM accompanied by HF.

In this short-term observation study, torasemide was shown to promote potassium excretion and drop of input and output to the same extent as furosemide, even though the patients were on the potassium-sparing diuretic spirolactone or potassium chloride tablets. However, torasemide has proven to be more effective in CHF with respect to reducing CRP and NTproBNP compared with furosemide, while in long-term follow-up, torasemide displayed a lower incidence of arrhythmia and better recovery of cardiac remodeling than did furosemide. There may be a potential dependent relationship between low incidence of arrhythmia and decrease in LVEDD. Torasemide displayed a better prognosis than did furosemide.

Our partial findings about lower mortality compared furosemide was identical as in the Torasemide in Congestive Heart Failure Study (Diez et al., 2009). Torasemide differs from furosemide since it has a longer half-life and longer duration of action, and it may block the renin-angiotensin-aldosterone systems by inhibiting aldosterone binding to its receptor. Therefore, it may reduce left ventricular remodeling. Aldosterone activity has been shown to promote myocardial fibrosis, potassium and magnesium depletion, sympathetic activation, parasympathetic inhibition and baroreceptor dysfunction. Anti-aldosterone treatment is recommended for the treatment of advanced heart failure (NYHA class III-IV). In vitro studies in which pharmacological concentrations of torasemide and furosemide were used, have shown that torasemide but not furosemide inhibits angiotensin II-induced vasoconstriction and vascular growth-promoting activity. The above can account for its ability for better recovery of LEVDD, resulting in lower incidence of arrhythmia and lower mortality in CHF patients. ACEI drugs also have biological effects of anti-angiotensin II to prevent heart remodeling. In other words, we can conclude that torasemide combined with ACEI has a superficial effect on LVEF compared with furosemide combined with ACEI, which provides reasons to widely use torasemide. Lower 
$\mathrm{Ca}^{2+}$ sensitivity in force generation is reported to exist in patients with HF symptoms or occurrence of sudden death in these DCM patients (Diez et al., 2009). Thus, it inspires us to further investigate the molecular mechanism underlying the lower mortality of torasemide.

\section{Limitation}

A limitation of the study was the short follow-up time. We therefore decided to investigate the therapeutic effect of torasemide for more than 3 years and to explore the potential molecular mechanism of lower mortality of torasemide.

\section{CONCLUSION}

DCM often coexists with cardiac symptoms and systemic symptoms, easily complicated with arrhythmia and heart failure, and its mortality is high. Occurrence rate of arrhythmia was closely related to heart function level. Torasemide with an anti-aldosterone effect was more effective against DCM with respect to reducing incidence of arrhythmia and attenuating symptoms of HF compared with furosemide.

\section{ACKNOWLEDGMENTS}

Research supported by the Beijing Municipality Natural Scientific Fund (\#7122168), the Introduced and Integrated Demonstration Projects of Hainan Province (\#YJJC20130009), and the Chinese PLA General Hospital Clinical Scientific Research Fund (\#2012FCTSYS-2024). We thank Dr. Xin Wei for design of the study and criticisms of the manuscript.

\section{REFERENCES}

Aleksova A, Merlo M, Zecchin M, Sabbadini G, et al. (2010). Impact of atrial fibrillation on outcome of patients with idiopathic dilated cardiomyopathy: data from the Heart Muscle Disease Registry of Trieste. Clin. Med. Res. 8: 142149.

Bakalli A, Kamberi L, Pllana E, Zahiti B, et al. (2010). The influence of left ventricular diameter on left atrial appendage size and thrombus formation in patients with dilated cardiomyopathy. Turk. Kardiyol. Dern. Ars. 38: 90-94.

Bielecka-Dabrowa A, Goch JH, Rysz J, Maciejewski M, et al. (2010). Influence of co-existing atrial fibrillation on the efficacy of atorvastatin treatment in patients with dilated cardiomyopathy: a pilot study. Lipids Health Dis. 9: 21.

Cosin J and Diez J (2002). Torasemide in chronic heart failure: results of the TORIC study. Eur. J. Heart Fail. 4: 507-513.

Dickerson JA, Raman SV, Baker PM and Leier CV (2013). Relationship of cardiac magnetic resonance imaging and myocardial biopsy in the evaluation of nonischemic cardiomyopathy. Congest. Heart Fail. 19: 29-38.

Diez J, Coca A, de Teresa E, Anguita M, et al. (2009). TORAFIC study protocol: torasemide prolonged release versus furosemide in patients with chronic heart failure. Expert. Rev. Cardiovasc. Ther. 7: 897-904.

Dimas VV, Denfield SW, Friedman RA, Cannon BC, et al. (2009). Frequency of cardiac death in children with idiopathic dilated cardiomyopathy. Am. J. Cardiol. 104: 1574-1577.

DiNicolantonio JJ (2012). Should torasemide be the loop diuretic of choice in systolic heart failure? Future. Cardiol. 8: 707-728.

Eigenthaler M, Engelhardt S, Schinke B, Kobsar A, et al. (2003). Disruption of cardiac Ena-VASP protein localization in intercalated disks causes dilated cardiomyopathy. Am. J. Physiol. Heart Circ. Physiol. 285: H2471-H2481.

Flevari P, Theodorakis G, Leftheriotis D, Kroupis C, et al. (2012). Serum markers of deranged myocardial collagen turnover: their relation to malignant ventricular arrhythmias in cardioverter-defibrillator recipients with heart failure. Am. Heart J. 164: 530-537.

Fujii B and Takami M (2008). Normalization of left ventricular function following cardiac resynchronization therapy: left 
bundle branch block as a potential etiology of dilated cardiomyopathy. Circ. J. 72: 1030-1033.

Ganiats TG, Browner DK and Dittrich HC (1998). Comparison of quality of Well-Being scale and NYHA functional status classification in patients with atrial fibrillation. New York Heart Association. Am. Heart J. 135: 819-824.

Gosselin-Badaroudine P, Keller DI, Huang H, Pouliot V, et al. (2012). A proton leak current through the cardiac sodium channel is linked to mixed arrhythmia and the dilated cardiomyopathy phenotype. PLoS One 7: e38331.

Grimm W (2012). Prophylactic implantable defibrillators in dilated cardiomyopathy. Herz 37: 859-866.

Han LN, Guo SL, Li TL, Ding GL, et al. (2013). Effect of immune modulation therapy on cardiac function and T-bet/ GATA-3 gene expression in aging male patients with chronic cardiac insufficiency. Immunotherapy 5: 143-153.

Haugaa KH, Goebel B, Dahlslett T, Meyer K, et al. (2012). Risk assessment of ventricular arrhythmias in patients with nonischemic dilated cardiomyopathy by strain echocardiography. J. Am. Soc. Echocardiogr. 25: 667-673.

Hay I, Melenovsky V, Fetics BJ, Judge DP, et al. (2004). Short-term effects of right-left heart sequential cardiac resynchronization in patients with heart failure, chronic atrial fibrillation, and atrioventricular nodal block. Circulation 110: 3404-3410.

Koutalas E, Kanoupakis E and Vardas P (2013). Sudden cardiac death in non-ischemic dilated cardiomyopathy: a critical appraisal of existing and potential risk stratification tools. Int. J. Cardiol. 167: 335-341.

Lourenço P, Paulo AJ, Paulo C, Mascarenhas J, et al. (2010). Higher C-reactive protein predicts worse prognosis in acute heart failure only in noninfected patients. Clin. Cardiol. 33: 708-714.

Mann SA, Castro ML, Ohanian M, Guo G, et al. (2012). R222Q SCN5A mutation is associated with reversible ventricular ectopy and dilated cardiomyopathy. J. Am. Coll. Cardiol. 60: 1566-1573.

McNally EM, Golbus JR and Puckelwartz MJ (2013). Genetic mutations and mechanisms in dilated cardiomyopathy. $J$. Clin. Invest 123: 19-26.

Nakagomi A, Seino Y, Endoh Y, Kusama Y, et al. (2010). Upregulation of monocyte proinflammatory cytokine production by C-reactive protein is significantly related to ongoing myocardial damage and future cardiac events in patients with chronic heart failure. J. Card. Fail. 16: 562-571.

Okutucu S and Oto A (2010). Risk stratification in nonischemic dilated cardiomyopathy: Current perspectives. Cardiol. J. 17: 219-229.

Pruszczyk P, Kostera-Pruszczyk A, Shatunov A, Goudeau B, et al. (2007). Restrictive cardiomyopathy with atrioventricular conduction block resulting from a desmin mutation. Int. J. Cardiol. 117: 244-253.

Richardson P, McKenna W, Bristow M, Maisch B, et al. (1996). Report of the 1995 World Health Organization/International Society and Federation of Cardiology Task Force on the definition and classification of cardiomyopathies. Circulation 93: 841-842.

Serio A, Narula N, Kodama T, Favalli V, et al. (2012). Familial dilated cardiomyopathy. Clinical and genetic characteristics. Herz 37: 822-829.

Slavich M, Florian A and Bogaert J (2011). The emerging role of magnetic resonance imaging and multidetector computed tomography in the diagnosis of dilated cardiomyopathy. Insights Imaging 2: 453-469.

Sugihara M, Odagiri F, Suzuki T, Murayama T, et al. (2013). Usefulness of running wheel for detection of congestive heart failure in dilated cardiomyopathy mouse model. PLoS One 8: e55514.

Sun LP, Wang L, Wang H, Zhang YH, et al. (2010). Connexin 43 remodeling induced by LMNA gene mutation Glu82Lys in familial dilated cardiomyopathy with atrial ventricular block. Chin. Med. J. 123: 1058-1062.

Suzuki T, Shioya T, Murayama T, Sugihara M, et al. (2012). Multistep ion channel remodeling and lethal arrhythmia precede heart failure in a mouse model of inherited dilated cardiomyopathy. PLoS One 7: e35353.

Tang Y, Tian X, Wang R, Fill M, et al. (2012). Abnormal termination of $\mathrm{Ca}^{2+}$ release is a common defect of RyR2 mutations associated with cardiomyopathies. Circ. Res. 110: 968-977.

Tsutamoto T, Sakai H, Ibe K, Yamaji M, et al. (2011). Effect of atorvastatin vs rosuvastatin on cardiac sympathetic nerve activity in non-diabetic patients with dilated cardiomyopathy. Circ. J. 75: 2160-2166.

Wei SR, Chen XS, Chen HF, Sun XP, et al. (2012). Expression of fas protein of myocardium in dilated cardiomyopathy. Fa. Yi Xue Za Zhi 28: 252-255.

Yoskovitz G, Peled Y, Gramlich M, Lahat H, et al. (2012). A novel titin mutation in adult-onset familial dilated cardiomyopathy. Am. J. Cardiol. 109: 1644-1650. 